\title{
A serious game for virtual rehabilitation: evaluation with patients and physiotherapists
}

\author{
Amanda M. M. Funabashi*1, Renan V. Aranha ${ }^{\dagger 1,2}$, Talita D. Silva ${ }^{\ddagger 1}$, Carlos. B. M. Monteiro ${ }^{\S 1}$, Willian S. Silva ${ }^{\llbracket 1}$, and Fátima \\ L. S. Nunes $\| 1,2$ \\ ${ }^{1}$ Laboratory of Computer Applications for Health Care (LApIS) - University of Sao Paulo \\ ${ }^{2}$ Polytechnic School of the University of São Paulo
}

\begin{abstract}
Games can make training procedures more engaging for patients. Considering the complexity of the process for upper limb function rehabilitation, this paper presents the development and an initial evaluation of the $\mathrm{AGaR}$ - a serious game with virtual reality and natural interaction, both to aid patients to execute repetitive exercises and to aid physiotherapists to follow the rehabilitation process. Additionally, we obtain and analyze data about patients' engagement as a differential in relation to others games developed for similar goals. In this game, the patient has to associate two different images with complementary meanings, using a movement sensor to drag the image to the target. We conducted an experiment with physiotherapists in order to evaluate the feasibility of applying the game in real therapies. We also conducted an initial experiment with patients. The results show that physiotherapists believe that the game is effective and might be used during therapies. From the experiments with patientss, we obtained that the number of wrong associations made by them varies according to patient, with no standard found. The engagement tends to increase during use of the game, throughout the rounds. Keywords: Serious Games, Virtual Reality, Virtual Rehabilitation, Gametherapy, Natural Interaction, Affective Computing.
\end{abstract}

\section{INTRODUCTION}

Stroke is the leading cause of long-term disability in the world [1] and upper limb paresis is one of the most frequent and persistent impairments following stroke. Only $12-34 \%$ of stroke patients fully recover the upper limb functioning, which seems to be required to use the affected arm in daily tasks [2].

In order to recover the upper limbs functions, rehabilitation programs include constraint-induced movement therapy [3] and electrical somatosensory stimulation [2], for example. However, games using Virtual Reality (VR) technology can be a great ally in this process. In special, Serious Games (SG) can be the leveraging of the power of computer games to captivate and engage end-users for specific purposes, such as to develop new knowledge and skills [4], attempting to create solid and relevant learning experiences for a wide variety of audiences [5].

Sometimes, the rehabilitation process can be wearisome and repetitive. During this process, recognition of the patient's emo-

*amanda.funabashi@usp.br

†renanvinicius@usp.br

$\ddagger$ ft.talitadias@gmail.com

$\S$ carlosfisi@uol.com.br

ฯwillian.severino.silva@gmail.com

" fatima.nunes@usp.br tional status can become an important information for the rehabilitation team. Emotions can play a critical role in the human brain, e.g., having influence in the rational decision-making, as well as in the perception and human intelligence [6]. Thus, the use of Affective Computing (AC) can be an important topic for improving human-computer interaction, increasing communication beyond human and computer [7]. AC is the observation and development of systems that can sense, interpret, procedure and answer to humans simulating affect, and empathy. Usually this area uses methods to recognize the six basic human emotions (fear, anger, happiness, surprise, disgust and sadness)[8].

According to [9], the use of VR and affective computing in the rehabilitation process of post-stroke patients can significantly contribute to improving the performance of day to day tasks. In [10] and [11], the benefits of using VR during rehabilitation programs are presented. Such benefits include greater motivation and commitment of patients, since the lack of adherence to therapy is one of the major barriers to rehabilitate individuals with disabilities [12].

In VR, both patient and therapist interact with a multidimensional, multisensory computer-generated environment that can be explored in real time. Moreover, VR offers the capacity to individualize treatment needs to each patient, while providing standardization of assessment and training protocols. This indicates that VR can favor an exclusive approach where therapy can be provided within a functional, purposeful and motivating context [10].

Low levels of interaction between patient and environment during therapy, as well as boredom, fatigue, lack of motivation and lack of cooperation in attending therapy, have been reported by many individuals as aspects that cause progress restriction in rehabilitation [13]. The development and incorporation of VR applications in rehabilitation may increase the possibility of stimulation and interaction with the virtual world, providing interesting, engaging, and more motivating tasks when compared to traditional repetitive therapy, without increasing demands of professionals time [10].

Considering the context previously presented, this article aims to present the definition, the development, and an initial evaluation of the AGaR (Association Game for Rehabilitation). AGaR is a VR serious game to provide a playful way to carry out the rehabilitation process of the upper limbs to support the recovery of post-stroke patients. The player executes the task of associating images that have complementary meanings, e.g., a key combined with a door lock. A sensor is used to captured the movements of the player and respond in the virtual environment (VE), so that the images can be dragged in the VE.

Two main points are differentials of this game (1) the use of AC to recognize the emotional status and the engagement of the patients, providing subsidies for the physiotherapist decision-making; and (2) inclusion of mechanisms to allow physiotherapists customize the game for each patient, changing the used images. Also, the game allows communication between the professional and the patient, by authorizing the play among the executed actions. 
This article is divided into the following sections: Section 2 presents concepts about SG and Virtual Rehabilitation, as well as the exploration of the post-stroke rehabilitation theme. Section 3 presents similar works. The techniques and the methods to develop this research are presented in Section 4. The experimental evaluation is presented in Section 5 and the results of the experiments can be found in Section 6, while the discussions are addressed in Section 7 and the conclusions in Section 8.

\section{BACKGROUND}

\subsection{Post-stroke rehabilitation}

Stroke still is one of the major chronic diseases [14] in the world. It is also considered one of the main causes of disabilities [15], resulting not only in persistent neurological deficits but also in upper limb dysfunction, which affects about $85 \%$ of stroke survivors, and less than half recover motor function within three months of poststroke. In addition, the level of recovery of the motor function has a great influence on the participation of people in community environments and on the quality of life [1].

Rehabilitation programs generally focus on tasks with a large number of repetitions, which is essential for promoting motor learning [16] and it is positively related to better motor recovery [1, 17]. However, conventional treatments are not as frequent and intense as required because of the cost and of the commitment of patients in rehabilitation programs that eventually become repetitive. Thus, with the increasing accessibility to computer-aided technologies and the rapid technological evolution, the possibility of using VR as a form of approach for treating people with physical disabilities, including stroke, has arisen [18, 19, 20, 21].

It worth emphasizing that VR tasks are easier to understand, are more interesting, allow tailoring tasks to patient needs and can be used at home, besides providing clinical feedback for better followup by the therapist [22] [23]. In addition, conclusions about the efficacy of VR in the rehabilitation of people with neurological diseases point to the benefits for improving motor performance, balance and motor learning [24] [25] [26].

\subsection{Serious Games and Virtual Rehabilitation}

According to [27], SG are the accepted term for games with an educational intent, being engaging while the learning can be implicit or explicit. They can apply several areas, such as military to provide safe mechanisms for training tasks to be performed in hazardous circumstances or that could be costly to perform in the real world.

Although there is no current single definition of this concept, the most widely agreed meaning for SG is that they are (digital) games used for purposes other than just entertainment, and it is argued that they can have positive impacts on the player's development of a number of different skills [28]. Games seems to enhance motivation and increase students interests when using simulation game activities to teach other than conventional teaching methods [29].

In [30] is stated that the hippocampus absorbs knowledge and information that is connected with positive emotions for it to be transfered to the cortex for further processing. Thus, to activate the hippocampus, the way those informations are transmitted should be somewhat pleasant to evoke the interest and positive emotions.

SG are also reaching areas in health care, such as therapeutic sessions, physiotherapy, psychotherapy and occupational therapy, which can benefit from the captivating character of video games $[31,32]$. These areas are using games due to its capacity to motivate the player, develop skills and distract patients in pain management [32].

Virtual rehabilitation have driven new and unique treatment methods to therapy areas that can benefit from video games. It consists in the use of VR during rehabilitation processes, in a way that simulates the real world environment through a computer where the patient can see themselves performing functional tasks. It has been recognized by researchers due to its potential therapeutic benefits [33].

VR can be an optimal tool for therapies that aim neuroplastic mechanisms in the nervous system, allow for mass practice and provide training in environment that might be impractical to recreate in the real world [34]. Also, training in VR can provide potential benefits such as the ability to increase the duration, frequency and intensity of the therapy that could be provided by using semiautomated programs and this technology allows rehabilitation at home instead of at rehabilitation center [35].

Furthermore, VR rehabilitation exercises can be made to be engaging so that the patient feels immersed in the simulated world and this is extremely important for the patient to feel motivated. VR can also provide distraction to the user during pain management, distress or unpleasantness during medical procedures. Investigators hypothesize that VR acts as a nonpharmacologic form of analgesia by exerting an assortment of emotional, emotion-based cognitive and attentional processes on the body. Although the exact neurobiological mechanisms behind the action of VR on the patient is still unclear, some investigations examine the complex neurobiological interplay of the brain cortical activity and the neurochemistry associated with immersive VR [36].

\subsection{Natural User Interfaces}

Natural User Interface (NUI) is an emerging human-computer interaction that focuses on human abilities such as touch, vision, voice, motion, and on higher cognitive functions, such as expressions, perception and recall [37].

There is a range of different relatively low-cost devices that can be used to infer mechanical motion with natural interaction. Some examples include Nintendo Wii Remote, PlayStation Move, and Microsoft Kinect [38].

NUIs do not require time for familiarizing to the management of any device such as mouse, joystick or keyboard; which can produce an unnecessary effort to establish a contact among users, the digital environment and the technology [39]. They replace conventional input by a command line interface or by interface devices with communication means that are closer to the way in which human beings interact; they give users the opportunity to interact with computers in a natural and intuitive way [40].

\section{Related Works}

The emergence of SG with VR and their use for various purposes, such as health care, is investigated in many studies. The literature presents the use of virtual rehabilitation for different problems and using various computational resources.

The authors of [41] developed a series of mini-SG using the Kinect sensor. They are gamified versions of conventional rehabilitation exercises that patients execute in therapy sessions, but using VR, making them less dangerous, providing more fun and customizable therapy routines.

Aiming at post-stroke rehabilitation, in [42] a game for hand movement recovery by using a glove as a way of interaction was developed. In this game, the patients have to clean a screen to reveal a pleasant image.

For the same purpose, low-cost robot-assistance is used in control games for training various tasks, using a platform that allows patients to adapt the therapy according to their needs [43]. In [44], a game to encourage gross movements was developed to improve the accuracy and speed of the upper limb movements, and also to improve visual discrimination and selective attention for post-stroke patients. According to the authors, initial results showed an impact on the recovery of movements both in the real world and in game performance. 
According to [9], VR systems apply relevant concepts for driving neuroplasticity, providing repetition, intensity and taskoriented training for paretic extremity, promoting motor recovery after stroke.

\section{Game conception AND deVELopment}

The AGaR SG was conceptually conceived to provide a playful rehabilitation process, using concepts of games and VR, in order to favor the process of rehabilitating upper limb function of poststroke individuals. To provide natural interaction, the motion sensor Kinect One was used as a NUI. It does not require the user to hold any device for its recognition. The game was developed by

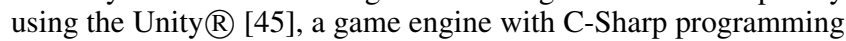
language.

\subsection{Conception}

The first step was defining of the requirements with physiotherapists researchers. Requirements included the game script and the playful elements that should be included, such as time, points, errors and discards.

In addition, to stimulate the player, making the activity more attractive, sound elements such as background music and sound effects were added according to the players answers, correct matches or errors.

\subsection{Storytelling of the game}

The game objective is to match a pair of images whose meanings are similar or complementary by associating them. The main scenario, illustrated in Figure 1, is composed of a central image as the target and four selectable images, out of which three are wrong, only one is correct. The correct one is randomly positioned among the four positions.

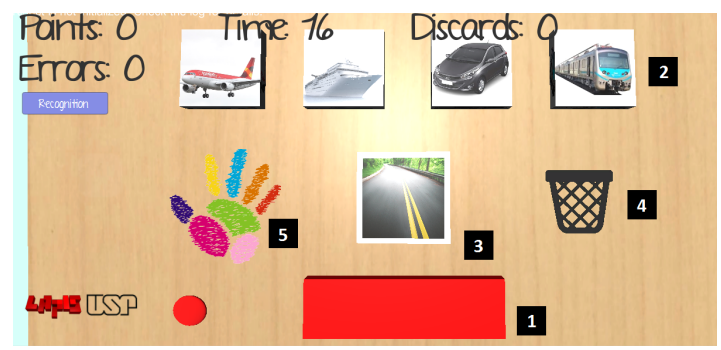

Figure 1: Main interface of the AGaR: Area 2 represents the selectable images, Area 3 represents the target image and Area 4 represents the discard target.

Located at the bottom of the screen and below the central image, there is a platform, the game starting point (Area 1 in Figure 1). Initially red, it becomes yellow when the player positions the cursor on it. When the physiotherapist authorizes the move, it turns green (Area 1 in Figure 3. The play can only be authorized if the player keeps the cursor on the platform and as long as the play is not released by the platform, the images are not selectable.

In the lower left corner, there is a red sphere that indicates if a image was selected, to facilitate the visibility of the game events. This sphere initial state is red and it turns green when an image is selected.

Furthermore, to discard an erroneously selected image, a trash bin was added to the right side of the screen. When an image needs to be discarded, the player can move it to the bin. Thus, this image will return to its initial position.

The game cursor is represented by an open hand (Area 5 in Figure 1) and it follows the movements performed by the hand of the player. The task is to move the correct picture to the central image, repeating this action the number of times determined by the physiotherapist. Figure 2 presents the state diagram of the game.

The execution of the game follows the steps bellow:

1. The patient positions the virtual hand on the platform;

2. when the platform turns yellow, the physiotherapist authorizes the move by pressing the space bar of the computer conventional keyboard;

3. when the move is authorized, the patient may place the virtual hand on one of the selectable images (Area 2 of Figure 1) to match the target image (Area 3 of Figure 1). If the patient has selected the wrong image, it can be discarded in the bin (Area 4 of Figure 1). In this case, the discarded image will return to its initial position, and a new image can be selected, repeating steps 1 and 2;

4. the patient drags the selected image to the target;

5. if the combination is a match, a sound is emitted demonstrating that it is correct, the target image receives a green outline (Area 3 in Figure 3) and the game moves to the next round, in which steps 1 to 4 are repeated. Otherwise, if the combination is an error, a different sound is emitted demonstrating the mismatch, the target image receives a red outline and the selected image is destroyed. The latter action provides the patient with a smaller amount of selectable images in the same round; and then, the patient continues playing by repeating steps 1 to 4 ;

6. the game ends when all the rounds determined by the physiotherapist have been performed.

At the end of each round, the following data are stored:

1. reaction time: how long it took the patient to leave the platform after the move was authorized;

2. time to select a image:how long it took the patient between the authorization of the move and the selection of an image;

3. displacement time: how long it took the patient to move the selected image from its initial position to the target image;

4. total game time: the total execution time for each patient to complete the game rounds.

5. valence metric likelihood: calculated by an affective computing Software Development Kit (SDK), this metric measures the user experience as negative or positive.

6. engagement results: also calculated by an affective computing SDK, this metric aims to get the patient expressiveness.

The stored data allows the rehabilitation professional to evaluate and to monitor the evolution of the virtual rehabilitation of the patient.

\section{EXPERIMENTAL EVALUATION}

After the game development, two types of users were defined for the AGaR experimental evaluation: physiotherapist and patient. Each of them has a specific relationship with the game. For physiotherapists it is important to understand the feasibility of this type of software in the rehabilitation processes as well as to compare the virtual rehabilitation with the traditional rehabilitation process. For patients it is important maintaining of the engagement in the activities while bringing evolution in the upper limb movement. 


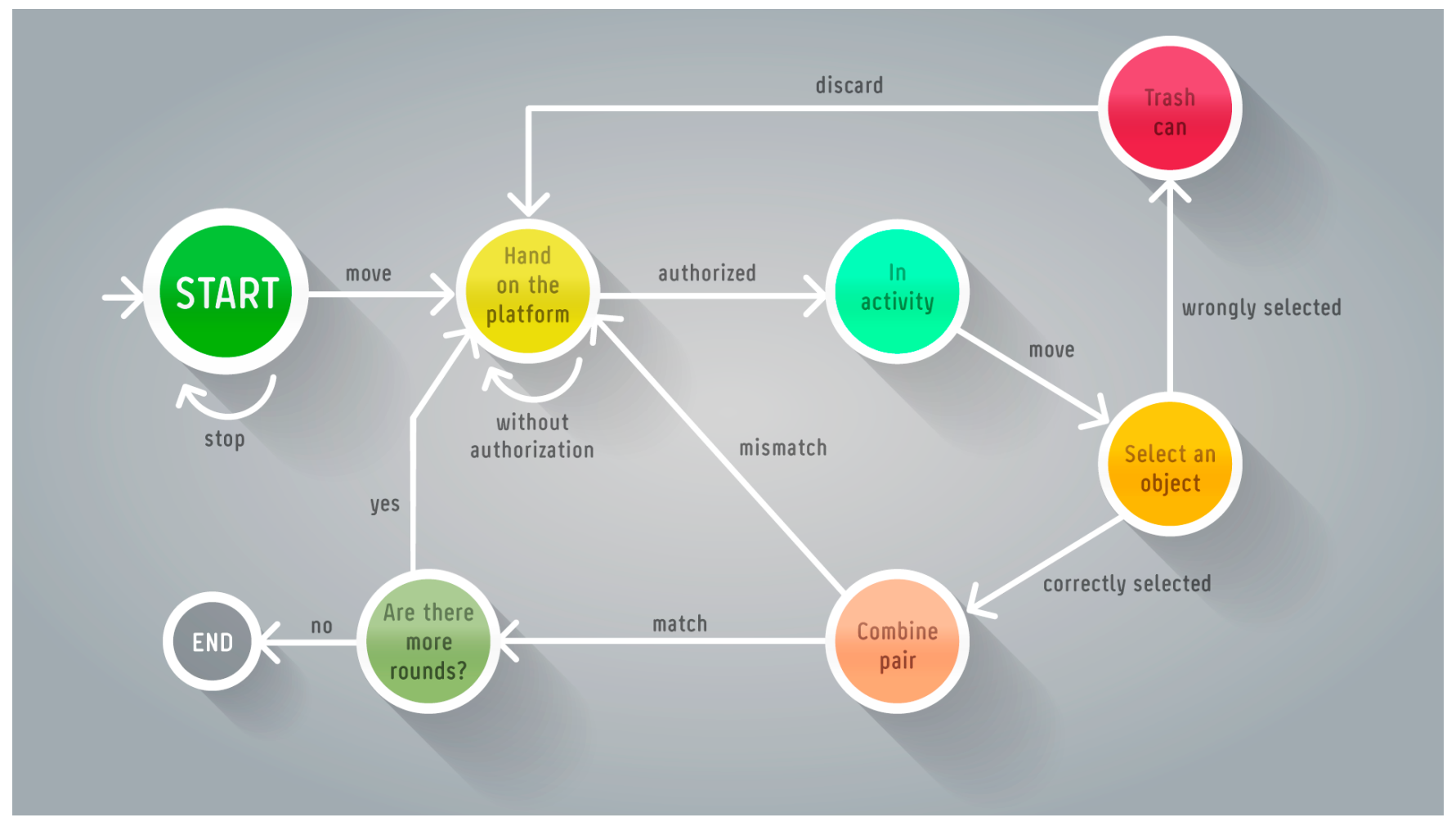

Figure 2: State diagram of AGaR.

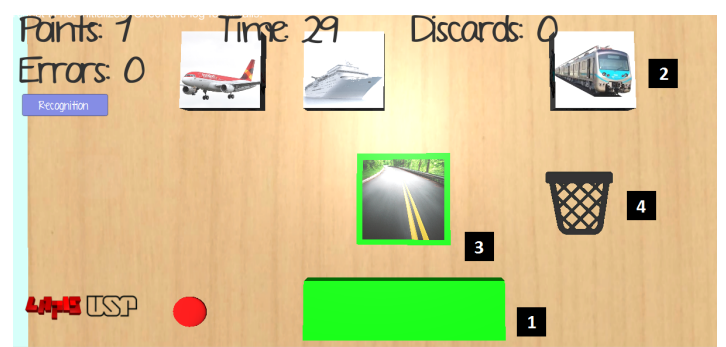

Figure 3: Interface of the AGaR where Area 3 is outlined with green color, demonstrating that the combination of the car with the road was correct.

\subsection{Experiment with physiotherapists}

In order to verify the acceptance and feasibility for using the game in real therapies, an experiment was carried out with physiotherapists. The aim was to analyze whether these professionals could use the game with ease, if they believed that the game could contribute to the improvement of patients' performance and whether such professionals would use it in rehabilitation processes.

In order to achieve the objective of the experiment, the following Research Questions (RQ) were defined:

- RQ1: Do the physiotherapists consider the use of SG and VR viable for rehabilitation?

- RQ2: Do the physiotherapists consider themselves capable of using VR programs with their patients?

- RQ3: Do the physiotherapists consider that the engagement and fun provided by VR games helpful in the process of rehabilitation?
- RQ4: Do the physiotherapists consider that the game can contribute to improve the performance of the patients?

- RQ5: Do the physiotherapists consider that the game could bring more benefits than the traditional rehabilitation process?

- RQ6: Do the physiotherapists consider that the game is easy to be used and does not require too much mental effort to be used during therapies?

To answer the questions RQ1 and RQ2, a self-efficacy questionnaire [46] was elaborated and then filled by the physiotherapists participating in the experiment in three stages: (1) before performing activities of the experiment, (2) after listening a brief explanation about the game working, and (3) after experimenting the game with virtual reality. The same questionnaire was applied to observe possible behavior changes after each activity.

A questionnaire for technology acceptance was used to answer the questions RQ3 to RQ6, filled by the participants in the end of the experiment. All questionnaires used a Likert scale for evaluation, being 1 for total disagreement and 5 for total agreement.

\subsubsection{Experiment Participants}

The experiment was carried out with ten physiotherapists researchers, which seven of them reported having five or more years of experience in clinical care for rehabilitation. The volunteers were asked about the use of SG during rehabilitation processes. Eight of them have already made use of digital games in rehabilitation, but $63 \%$ of them use this resource less than 2 years, as presented in Figure 4.

\subsubsection{User Interactions}

The experiment was conducted individually in a controlled environment. After signing a consent form, the participants were invited to 


\section{Experience using games}

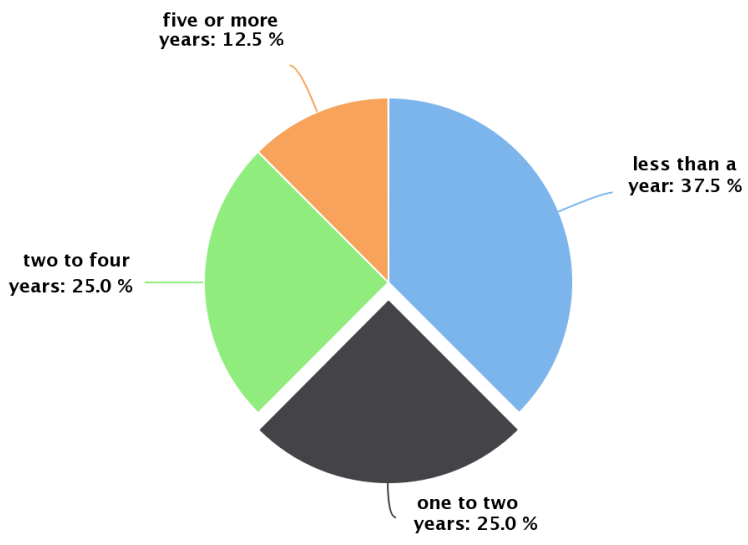

Figure 4: Experience of the physiotherapists using games during rehabilitation processes

respond to the Physiotherapist Profile Characterization Form, containing questions about degree of education, experience in attendance and prior use of Virtual Reality. They also answered the Physiotherapist's Self-Efficacy Form. After answering both questionnaires, an explanation was provided about the game's working so they could respond again to the Physiotherapist's Self-Efficacy Form based on what was presented. Then the participants were invited to play the game as they were patients (Section 5.2) to realize the gameplay of AGaR and the use of VR, as well as draw their conclusions about the use of games in rehabilitation process. After playing the game, the participants were requested to answer again the Physiotherapist's Self-Efficacy Form and the Physiotherapist's Technology Acceptance Form.

\subsection{Experiment with patients}

The AGaR evaluation was performed by an experiment in a controlled environment. The experiment was planned together with experts, who determined the number of rounds as well as the repetition of images during the sessions. Sessions were conducted by a physiotherapist specialized in clinical neurology, with experience in the use of Kinect sensor, who was previously trained in the application of the proposed protocol. Figure 5 exemplifies a session of the experiment.

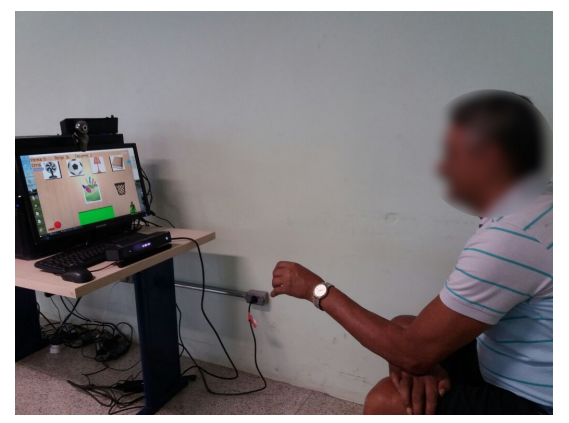

Figure 5: Experiment being conducted with a patient.

The protocol was composed by two parts: a pre-test session and a session with pre-determined amount of rounds to collect data about the movement performed by the patient.

In the pre-test session, the patient performed three tasks related to the protocol, aiming to clarifying doubts about the capture of the movements by the sensor and about the movement of the virtual hand.

In the second part, patients executed a sequence of rounds with different images. The images used in the game was chosen by the physiotherapists in a preset sequence in order to evaluate the improvement of the patient, avoiding the influence of the patient cognition process in the results, since the goal was to analyze the movement.

The sequence of sessions was divided in three cycles, resulting in thirty three rounds in total:

1. seven sets of three rounds were used. The first round has a car (the answer), a train, a ship and an airplane as selectable images and a road as the target image. The second round has a fish (the answer), a horse, a dog and a bird as selectable images and a aquarium as the target image. The third round has a ball (the answer), a book, a lampshade and a fan as selectable images and a soccer field as the target image;

2. those rounds explained above are repeated two times each one (the first round is repeated two times, the second round is repeated two times and so on)

3. the sequences from the last cycle repeats again, but this time the answer image changes: for the first round, instead of a car it was used a motorcycle; for the second round, instead of the used fish, it was chosen another specie of fish; and for the third round, instead of a ball, it was used a soccer stand.

\subsubsection{Experiment Participants}

All the participants in the experiment suffered stroke over six months. They were also right-handed and had no previous experience with virtual games.

The patients had paresis of the upper limb with distal predominance and they have an active wrist movement in extension above ten degrees and abduction/flexion of the shoulder above sixty degrees.

\subsubsection{Hardware and Software}

For the experiment we used a computer with Intel(R) Core(TM) i7 processor, 8.0 GB of RAM memory with Windows 8,64 bits and NVIDIA GeForce GT 430 graphic card.

The NUI device used in the experiment was the Kinect Sensor for Xbox One [47].

For the analysis of the participant's emotion, we used an emotion-sensing and analytics SDK by Affectiva [48] that just required a webcam to capture the image which is used to detect facial expressions. The SDK algorithm identifies some key landmarks on the face and, with machine learning, classify them into facial expressions and mapped the emotions based on the combinations of those expressions in real time.

\subsubsection{User Interactions}

The participants were placed seated about one meter and fifty centimeters from the motion sensor, with the monitor at eye level of the patients. The physiotherapist followed the entire intervention protocol, observing if the game presented some type of failure in capturing movement or selecting the images.

The participant did not need to manipulate any device, the realization of the activity was just by using their natural upper limb natural movements. The participant's upper limb used in the activity was the less affected by stroke, due to the facility in moving the virtual hand.

During the protocol application, the participant received verbal instructions to place the hand in the platform for the play to be released. If the patient missed the test, it would be restarted by placing the hand in the platform again. 
The participants carried out the protocol without interruptions in an environment that favored their concentration in the task. Each participant executed the activity once, completing the thirty three rounds.

\section{Results}

\subsection{Experiment with physiotherapists}

As mentioned, we defined some Research Questions in order to evaluate the opinion of physiotherapists about our approach. Following we presented the results according to these questions.

\subsubsection{Self-Efficacy Perception}

To answer RQ1 and RQ2 presented in Section 5.1, the Self-Efficacy Form was used to compare the physiotherapists perception about their abilities to use the game and their notion about the use of SG and VR during rehabilitation process before and after the execution of the experiment. Figures 6,7 and 8 show the results for the three questions presented in the form, which of them shows the results for each participant in this experiment.

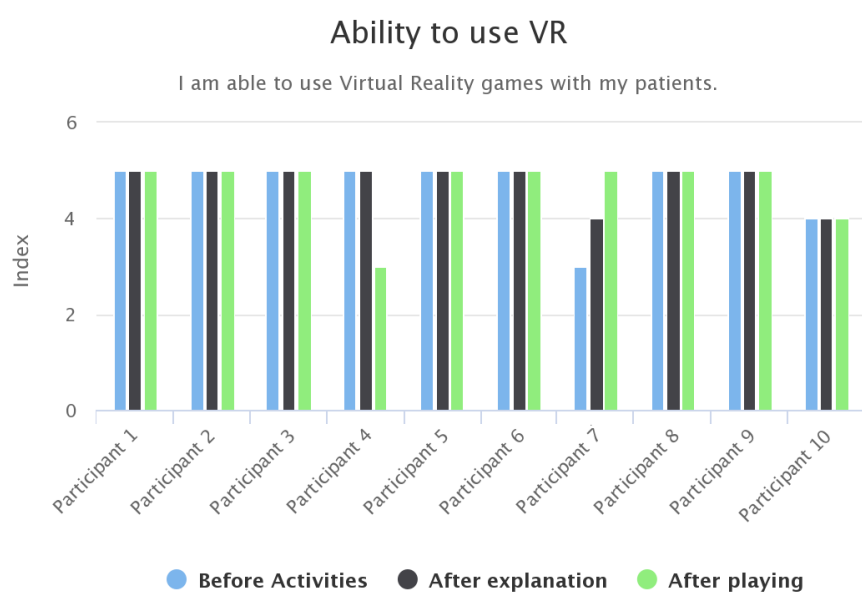

Figure 6: Physiotherapists opinion about their abilities to use VR with their patients before and after the experiment activities

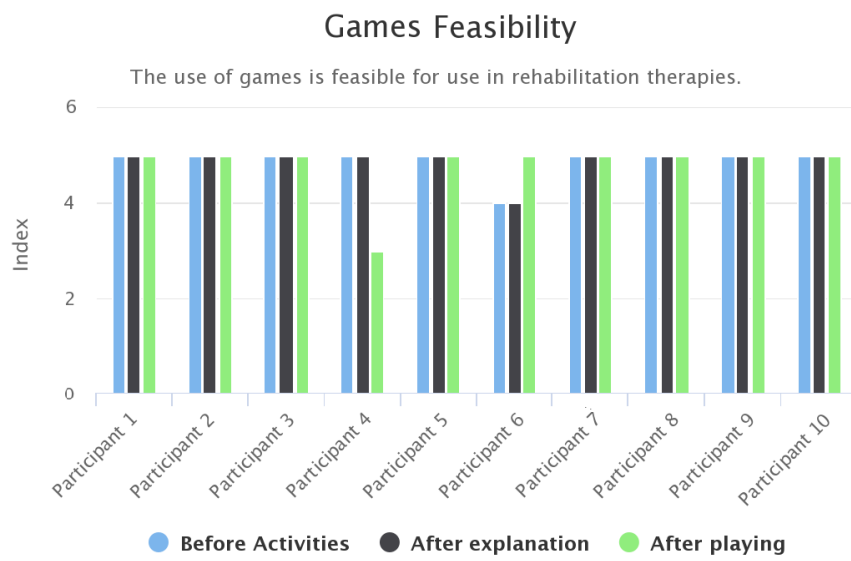

Figure 7: Physiotherapists opinion about the feasibility of using games during therapy processes before and after the experiment activities

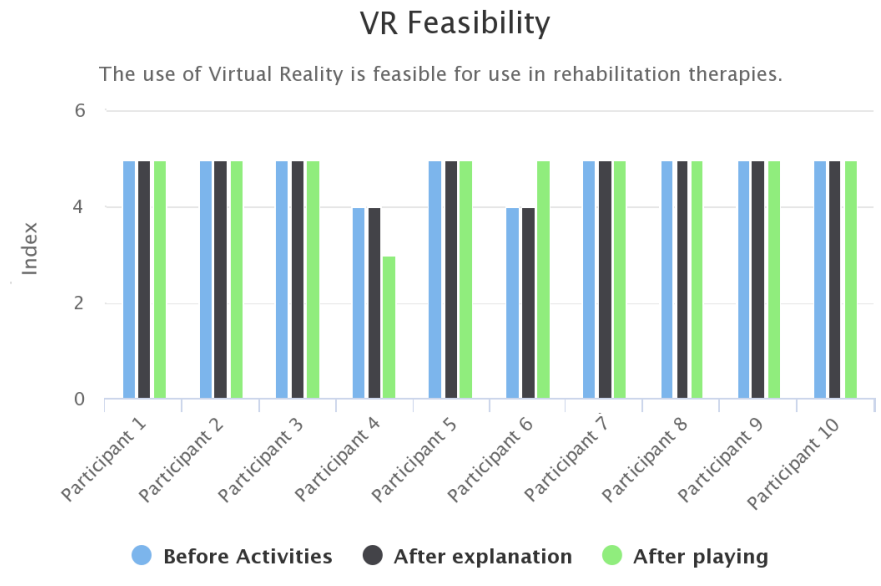

Figure 8: Physiotherapists opinion about the feasibility of using VR during therapy processes before and after the experiment activities

Most of the participants agree that they are able to use the game as well as that the use of VR and games are feasible in rehabilitation processes. One participant (Participant 7) did not fully agree with his ability to use the game, but after the experiment he changed his opinion. This also happened with Participant 6 , that firstly did not fully agreed with the feasibility of using VR and SG in rehabilitation, but had the opinion changed after playing the game. This improvement can be an evidence that the game provided a good experience and that they could visualize its use in their rehabilitation activities routine.

Another highlight that can be noticed was a decrease in agreement for Participant 4, who firstly agreed on her ability to use the game as well as the viability to use VR and SG during rehabilitation processes and after playing the game she disagreed. This could evidence some difficulties that the physiotherapists may find while using VR and SG in rehabilitation.

\subsubsection{Technology Acceptance}

To answer the questions RQ3 to RQ6 presented in Section 5.1, the Technology Acceptance Form was used to analyze how useful SG and VR can be for the rehabilitation process. The results are presented in Figure 9.

Almost all the participants fully agreed that the engagement provided by VR and also SG can assist their patients in rehabilitation, making this process less tedious and maybe encouraging them to continue the treatment. Also, all of them fully agreed that the use of VR and games might increase the patients' engagement to perform the rehabilitation activities.

The participants agreed that the game is useful and effective to be used in rehabilitation and all of them fully agreed that the game might contribute to the improvement of their patients performances. That indicates that AGaR can be used in rehabilitation, although some improvements in the game must be made to make it more adjustable to each patient and their own conditions, such as distance, speed and images.

According to the results presented, half of the participants fully agreed that the use of $\mathrm{AGaR}$ could bring more benefits than the traditional rehabilitation process, but none of them disagreed. That is an evidence that the physiotherapists professionals are tending to prefer the use of new technologies in the therapies due to the tendency of games to be more pleasurable.

Although the results show a positive evaluation of the game, the participants did not fully agreed that the game is easy and that does not require a lot of mental effort. Some of them reported during the experiment the lack of precision of the NUI device that could 


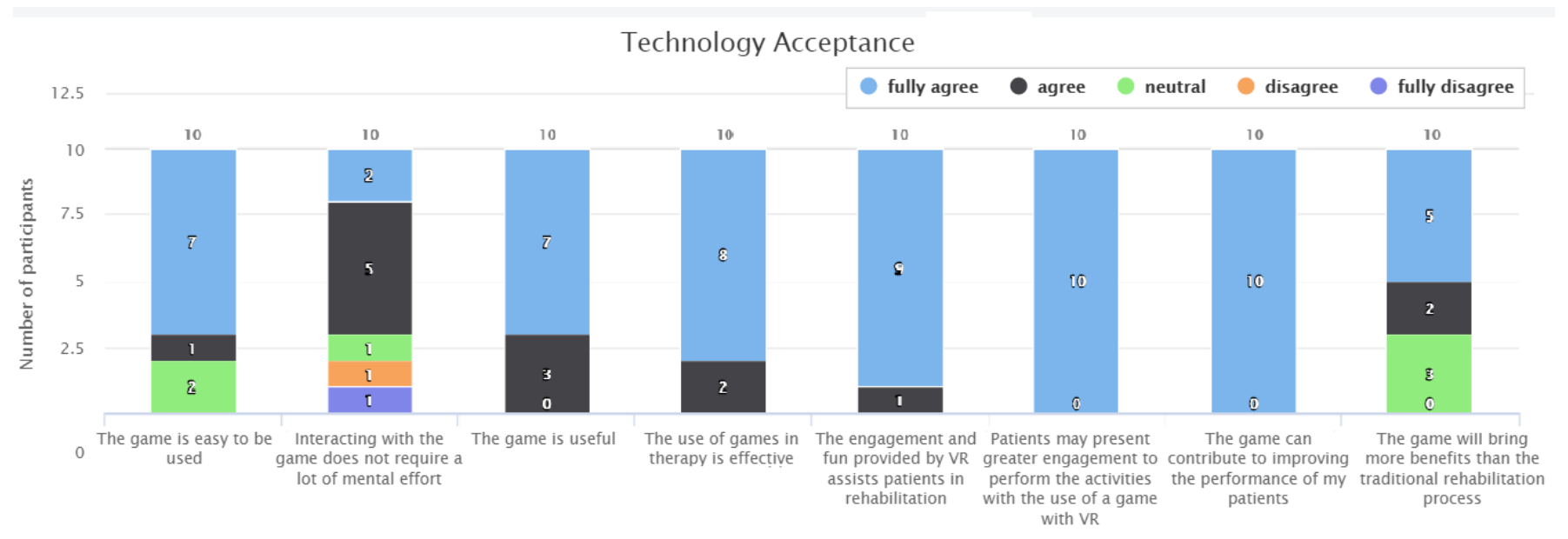

Figure 9: Technology Acceptance

make the game require more effort to be used when compared to the traditional process.

\subsection{Experiment with patients}

Four patients accomplished the protocol previously mentioned. We analyzed the results considering three dimensions: the sensor, the effectiveness of the AGaR and the facial expressions, which provide us insights about the emotional status of the patients.

\subsubsection{Sensor evaluation}

To evaluate the sensor, we analyzed the errors and discards executed by the patients, as well as observations of the physiotherapist who conducted the experiment. Considering that the task does not require high rate of comprehension and attention, our hypothesis is that the number of discards and errors should decrease throughout the sessions. Thus, a variation of these variables could be related to the accuracy of the sensor.

Figure 10 presents the number of discards of all participants and Figure 11 presents the number of errors committed by the participants. To evaluate the variables mentioned above, the graphs were divided per cycle, having the first one seven repetitions of three rounds; the second one has two repetitions of the same three rounds; and the third cycle has two repetitions of the three rounds with different images answers, as previously presented.

The full lines in both graphs represents the number of errors or discards performed in each sequence of rounds. The dotted line represents the linear tendencies of those numbers, verifying if those numbers tends to reduce or to increase.

It is possible to observe that there is no pattern in the number of discards made by the participants. While patient 1 and patient 4 times tend to decreases the amount of discards, patient 2 and participant patient 3 tends to increase them.

On the other hand, the number of errors increase for all the participants except for participant patient 2, that had committed no errors.

Additionally, the professional that followed the experiment noticed that instead of discarding the image, some patients sometimes committed an error, dragging the selected image to the target one. Thus some errors can actually be considered as a discard.

These graphs can indicate that the use of the Kinect sensor may not have been entirely effective, as the number of discards and errors committed by the participants throughout the game should have decreased, as the rounds repeats in cycles. The increase of those numbers or even the variation of these variables can be an evidence

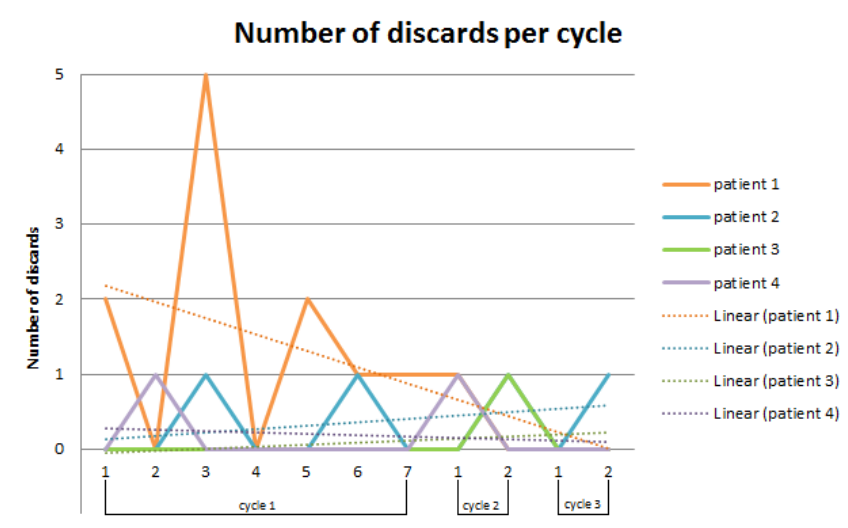

Figure 10: Number of discards made by each participant per cycle

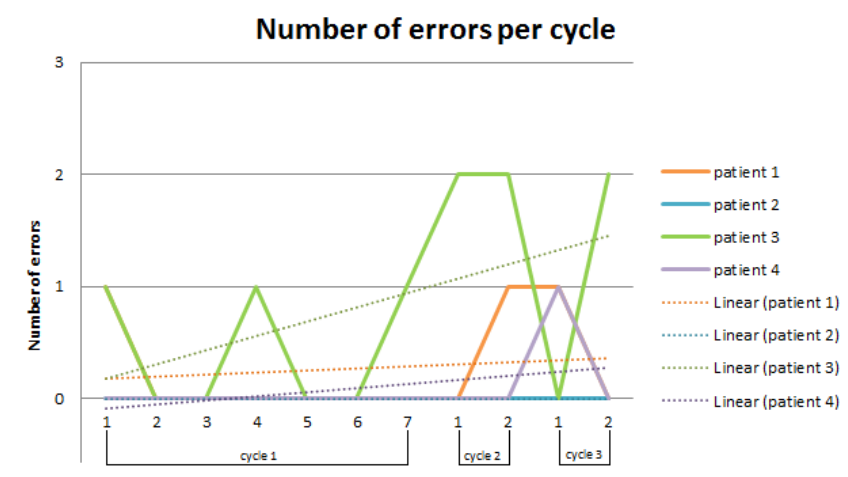

Figure 11: Number of errors committed by each participant per cycle

that the participants could not make the correct movement due some failure in the capture of their upper limbs.

\subsubsection{Effectiveness of the game}

To evaluate the effectiveness of the AGaR in the rehabilitation process, we analyzed the timing data collected for each patient indi- 
vidually: output time (reaction time), selection time and hit time, considering each round of all the thirty three performed. Figure 12 show these data. All the rounds are shown instead of only the cycles in order to obtain a better and more precise analysis. The dashed lines represent the average time of each variable (output time, selection time or hit time) for each patient. The dotted lines represent the linear tendency of each variable, that was chosen to analyze what tendency does these variables take throughout the rounds, e.g., if they tend to increase or decrease linearly.

We considered that finding the correct image become easier throughout the rounds, since the protocol was established to decrease the cognitive load required as the game progresses, and the used images repeats. Thus, in this analysis, our hypothesis is that if the AGaR is effective, then those variables related to time should tend to decrease throughout the rounds. Additionally we relate timing data to errors and discards presented in the previous section, in order to infer how was the effect of the AGaR for each patient.

Figure $12 \mathrm{~A}$ present the times for patient 1 . In this case, the hit time tends to decrease. Additionally, from the graphs in Figure 10 and Figure 11, we observe that the number of discards of this patient 1 tends to decrease while the number of errors remains almost stable, with only two errors at the end of the game. From information provided by the professional responsible for the experiment, we realized that some discards could have occurred instead of some errors.

Thereby, considering the set of information, there are indications that this participant could have learned the movement or it became automatic for the participant to perceive the answer faster, as the images sequence repeats. Another indication for that hypothesis is the decreasing selection time, that can be an evidence of the movement learning.

Figure 12B presents the time variables for patient 2 . The graph shows that the hit time remains almost constant (see the tendency line of this variable in the green colored dotted line). Also, the selection time tends to decrease throughout the rounds, which can be an evidence of movement learning or that the perception of the patient became faster, making a faster movement possible. However, the number of discards presented in Figure 10 tends to increase for this participant, which can indicate a wrong movement, selecting an undesired image. That can be an evidence that the increasingly number of discards may have been caused by the faster movement. Furthermore, based on the number of errors (Figure 11) this participant committed no errors, indicating that the answer was known by him.

Timing variables of patient 3 is presented in Figure 12C. There was an almost constant hit time during the game, which can be realized by the tendency line for hit times. For this patient, the tendency line of the selection time tends to increase throughout the rounds. This can be an evidence that the game may not have been effective for this participant learning. Another indication for that is the drastic increase of number of errors committed by this participant (Figure 11) and also the slightly increasing number of discards (Figure 10). With the information given by the professional that followed the experiment, we can consider that some of those errors may have been a discard. As the previous patient, these data can indicate that the movement could not be learned by the participant, as the number of discards increases and the patient could not select the correct image and had to discard it.

Figure 12D shows the timing data of patient 4. According to the hit time tendency line, we can observe that the hit time maintains almost constant and, with the selection time tendency line, that the selection time tends to increase. Analyzing Figure 10 and Figure 11 for this participant, we see that the number of discards tends to decrease while the number of errors tends to increase. Considering the information given by the experiment conductor, some of these errors can also be classified as discards, so it is possible to infer that the number of discards may increase instead. Therefore, it can be an evidence that the game was not very effective for this participant learning, as this increasingly number of discards might indicate that the movement to select an image was wrong and the participant had to discard it.

\subsubsection{Emotion Analysis}

In order to analyze if the AGaR was engaging, the results of valence and engagement given by the Affectiva's SDK were used. According to Affectiva, the valence is a measure of the positive or negative nature of the recorded person's experience, while the engagement is the measure of facial muscles activation that illustrates the person's expressiveness[48].

To calculate the valence metric likelihood, a set of facial expressiveness is used. To increase positively its likelihood, smiles and cheek rises are detected in the facial images. To increase negatively the valence's likelihood, the following changes in the human face's areas are considered: inner brow raise, brow furrow, nose wrinkle, upper lip raise, lip corner depressor, chin raise, lip press and lip suck. The range of values is from -100 to 100 , the more negative the values, the more negative is the patient's experience and the more positive the values, the more positive is the patient's experience. Besides, the engagement is calculated by the weighted sum of the measures of brow raise, brow furrow, nose wrinkle, lip corner depressor, chin raise, lip press, lip pucker, mouth open, lip suck and smile. The range of values is from 0 to 100 , the higher the value, the greater is the engagement.

The emotion experiment was performed with two of the participants: patient 2 and patient 4 . The graphs presented in this section are used to analyze if the game could promote engagement in the participants of the experiment. The full line of the graphs represents the engagement and valence measures given by the SDK throughout the game rounds, while the dotted line represents the tendency of these data, if they tend to increase or decrease linearly.

The valence results for patient 2 is presented in Figure 13B. The graph shows that the values are not standardized, with the tendency line being near the zero line, but still positive. According to the Affectiva's valence definition, this can indicate that this patient experienced both positive and negative moments during the game play almost equally.

Figure 13A shows the engagement results for patient 2. This graph shows that this patient presented expressiveness during the game and it slightly increased over the game play, according to the tendency line. Analyzing this graph together with the graph presented in Figure 13B of the valences results, it is possible do infer that the game, even with negative peaks, was engaging for this participant.

The valence results for patient 4 is presented in Figure 13D. For this patient, there was some negative peaks that may have been caused by an error or a difficulty in the recognition of the answer. However, the tendency line indicates that the valence metrics likelihood tends to increase. In other words, it can be an evidence that the participant had a positive experience in the game.

Engagement values for patient 4 is presented in Figure 13C. It shows high engagement levels that, according to the tendency line, increases throughout the game. This, along with the graph presented in Figure 13D may indicate that, for this participant, the game was more engaging and pleasurable to be played.

\section{Discussion}

As shown in Figures 6, 7, 8 and 9, positive results were obtained in the experiment with the physiotherapists. Most of them believe that VR and SG are useful and effective in rehabilitation and that they can contribute with patients' performance, but the total replacement of the traditional treatment by the use of VR and SG needs to be further studied. 
Times for Patient 1

A)

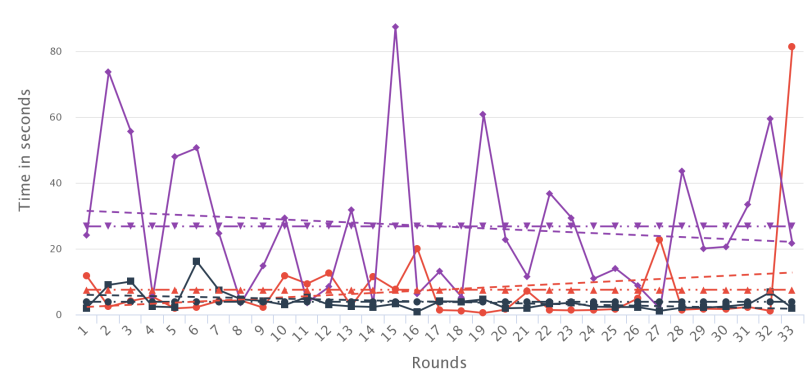

Times for Patient 3

C)

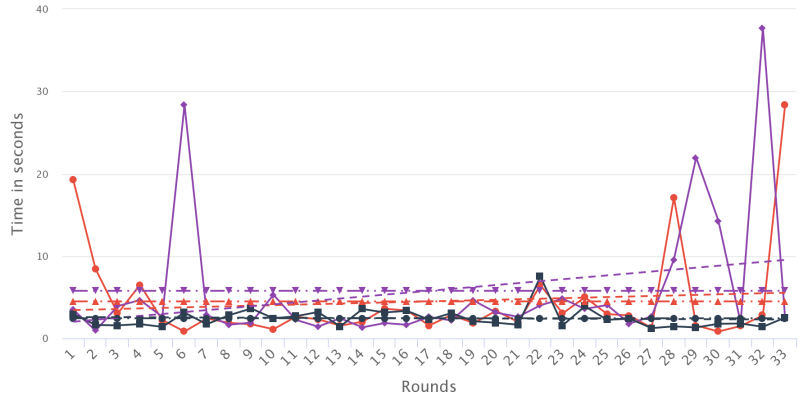

B)

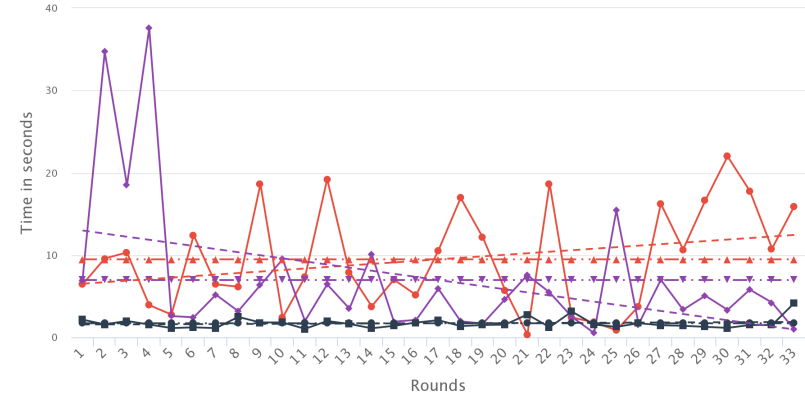

D)

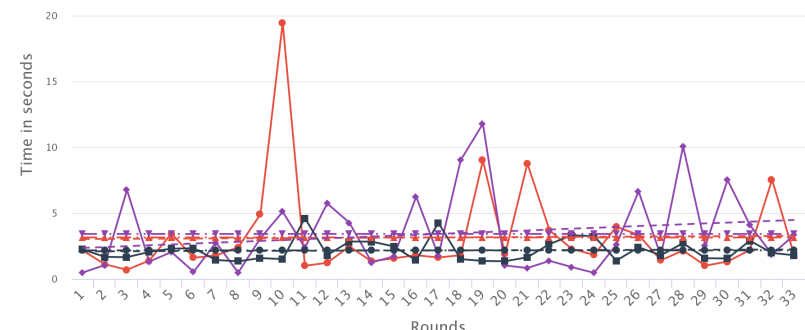

Times for Patient 2

Times for Patient 4

Figure 12: Game times for the four patients

Some remarks made by the physiotherapists that participated in the experiment referred to the adjustment of the game for different patients, making the speed faster or slower and the range bigger or smaller depending on the ideal treatment that the patient might have. For this, a calibration mechanism may be used to get how far the patient can reach and also how fast he can reach some point.

The physiotherapists also presented some complaints about the image repetition, so the rounds explained in Section 5.2 might be changed for the game not to be wearisome and repetitive.

Regarding the effectiveness of the AGaR considering the initial evaluation conducted with patients, we found positive results for patient 1 and patient 2, from the analysis based on their hit and selection times (which presented a decreasing tendency for both values) and also took into account the number of discards and errors made by them. Besides, for patient 3 and patient 4, the results did not evidence effectiveness of the game on their movement learning, since in general we did not find decrease in the times wasted to execute movements.

However, the analysis of the valence and engagement made with patient 4 indicates that, even though the results do not show clear effectiveness on his movement learning, the participant showed a high engagement and valence metrics. This can indicate that the game was engaging and that it may have been pleasurable to play, and, thus, it can be an alternative treatment for upper limp rehabilitation.

It is important to highlight that the data here presented was used to initially evaluate our game as a concept proof. It would be premature any definitive statement, since a greater number of sessions with these patients, considering a longer period of time, would be necessary to statistically prove any assertion. Additionally, characteristics of the patients should be deeper analyzed in order to relate their engagement with the game features. However, the concept proof shows that the storytelling, as well as the playful elements can provide effectiveness and engagement for some patients.

Although it was not possible to establish a direct relation between the emotion results and the performance in terms of game times and rates of the two patients, the graphs presented in Figure 13 show that this game can be engaging for the patients and can also be more motivating than the traditional process of rehabilitation. For a more accurate analysis of this relation, a experiment with more rounds, more sessions and more patients is necessary.

An additional remark concerns to the behavior of the Kinect sensor in relation to its fidelity to detect real movements to reproduce in virtual environments. Although we discussed that the precision of this sensor seems limited and sometimes can have induced some errors in the patients' performance, most of the times we considered it worked in a adequate way.

This indication is due to the fact that there is a direct relationship between timing variables and distances within virtual environment. The distance that the virtual hand must go to select an image is greater than the trailing distance between the selection and the association, being proportional to the distance trailed in real environment. It can be noticed in the participants graphs in Figure 12, as the average hit time is always lower than the average selection time.

Some physiotherapists also reported some lack of precision when playing the game using Kinect sensor during the experiment to select and grab a image to the corresponding place. This might be a problem when using the game during the therapy, as the patient may not do the right movement or feel stressed by making mistakes and discards that did not need to be committed. Other sensors such as Leap Motion [49] can be used, mainly if it is need for the patient 


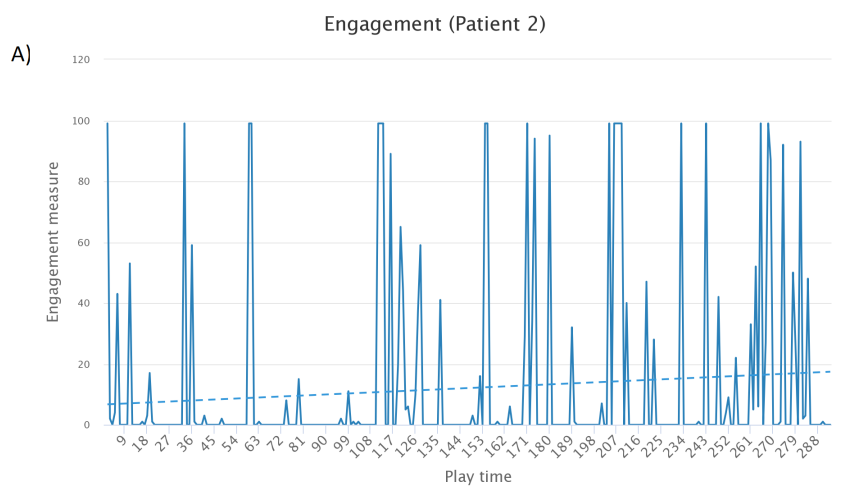

C)

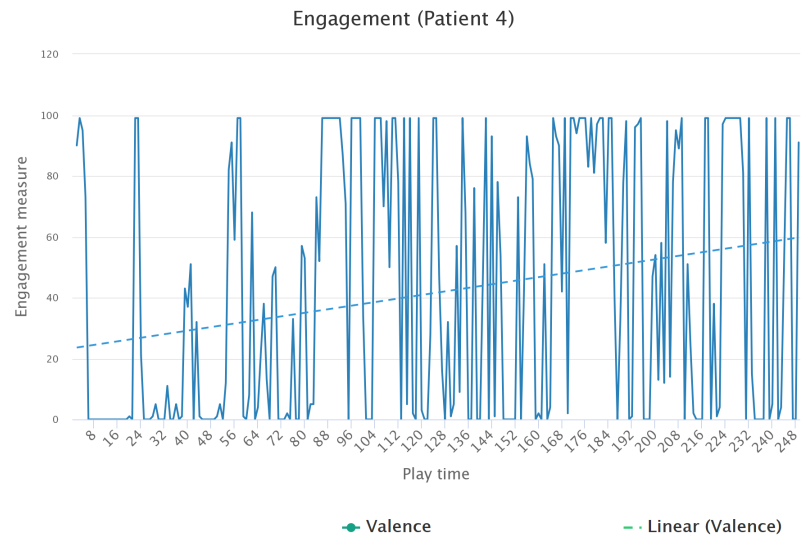

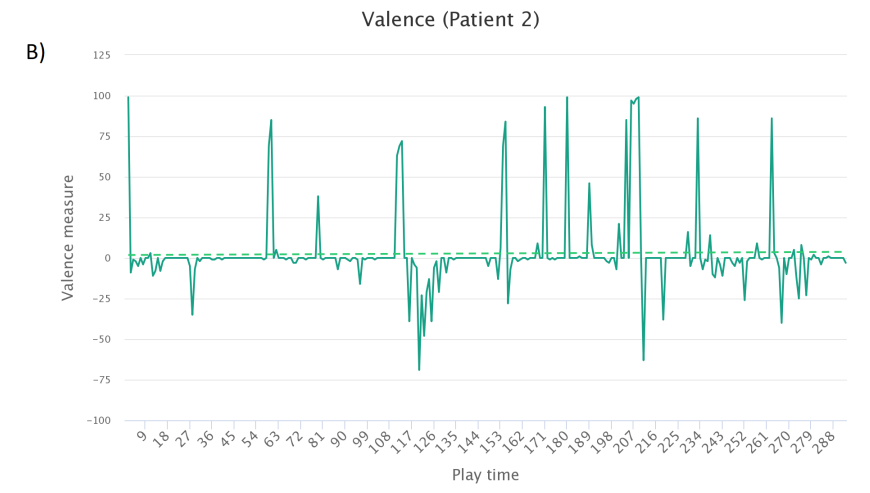

D)

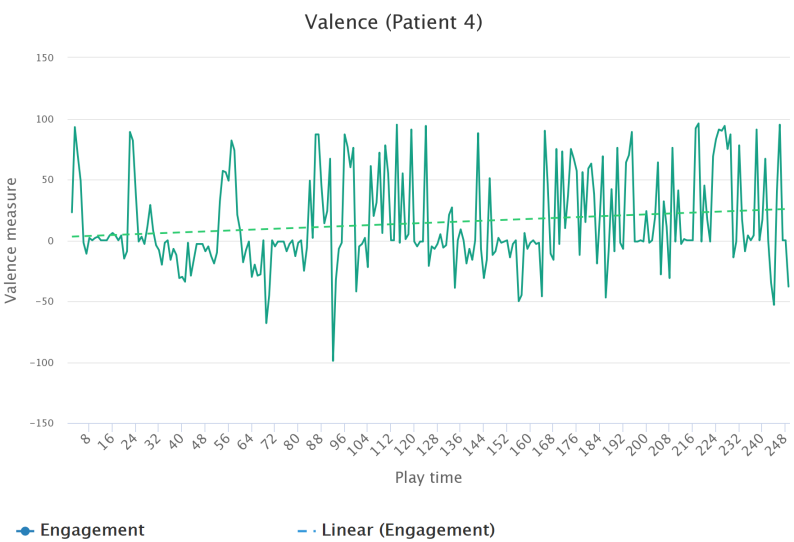

Figure 13: Engagement and valence values for patients 2 and 4

to practice fine motor control, as the sensor can capture fine hand and finger movements, as explored in [50], being more precise. In [51], the authors also explored the use of Leap Motion sensor for stroke rehabilitation; according to the authors, none of the participants of the experiment experienced any adverse effects. Furthermore, this study presented results that indicate that Leap Motionbased VR training not only facilitates the motor function recovery of paretic upper limbs, but also promotes neural reorganization.

Besides, the necessity to have a device may limit SG usability as the patient need to buy the sensor to use it at home. An alternative option can be the use of smartphones as motion sensor, as presented in [52], attaching the smartphone to the patient's hand. Also, the smartphone camera might be used with the real-time 3D gestural analysis, provided by frameworks, such as the ManoMotion framework, [53] to capture the patient's movement.

From the considerations above mentioned, we intend to extend the experiment with a greater number of patients to obtain more data to confirm the behavior of the sensor. More sessions and more patients must be considered to provided a more detailed analysis. As the sessions are short, with a few rounds to be played, only one session may not be enough to evaluate correctly the benefits of AGaR. Also, some participants related some cognitive difficulty on recognizing the answer for some rounds, or that the images were small. So that might have impaired the results accuracy, which requires review of the protocol to correct eventual failures.

\section{Conclusions}

As a proposal to bypass limitations that some patients face during the motor rehabilitation process, this work presented the use of Virtual Rehabilitation techniques in the process of upper limb motor rehabilitation. This investigation is justified by the motivation to find alternative treatments that can increase the patients engagement during the rehabilitation process, contributing to the reduction of the evasion rate from rehabilitation centers.

In this investigation, a serious game named AGaR was developed using VR techniques for upper limb rehabilitation. This game provide a VE that allows the execution of association tasks, where players have to link two images that have complementary meanings. The results show that the game was effective for some participants to improve their upper limb movement, but for more concrete evidences, a greater number of sessions is needed, as well as a greater number of patients. Also, the experiment carried out with physiotherapists got positive results about the effectiveness of the game to be used in rehabilitation processes. Additionally, we think this game can be improved by considering an evaluation of the initial conditions and limitations of each patient on the beginning of the experiment. This could provide information to compare the patient's evolution, which was not considered in the analysis here presented.

New features are planned for the next versions of this game, such as making available for the physiotherapist to position the images and to make possible for the professional to adjust the speed and sensibility of the sensor in a friendly interface. We also intent to make possible a previous calibration of the movement that each patient is able to perform in order to provide automatic adaptation of the game to the patient's conditions. This can be helpful to make the treatment more adjustable for the patients' need. Also, a more accurate analysis in the engagement and valence recognition using $\mathrm{AC}$ is planned. 


\section{ACKNOWLEDGMENTS}

The authors thanks Rumen Filkov for the free availability of the Kinect and Unity integration package as well as the volunteers who made the described experiment possible. The first author also thanks the support of the Ministry of Education Tutorial Education Program (PET).

\section{RefERENCES}

[1] T. J. D. Tomić, A. M. Savić, A. S. Vidaković, S. Z. Rodić, M. S. Isaković, C. Rodríguez-de Pablo, T. Keller, and L. M. Konstantinović, "Armassist robotic system versus matched conventional therapy for poststroke upper limb rehabilitation: A randomized clinical trial," BioMed Research International, vol. 2017, 2017.

[2] E. Ghaziani, C. Couppé, C. Henkel, V. Siersma, M. Søndergaard, H. Christensen, and S. P. Magnusson, "Electrical somatosensory stimulation followed by motor training of the paretic upper limb in acute stroke: study protocol for a randomized controlled trial," Trials, vol. 18, no. 1 , p. $84,2017$.

[3] H. Seok, S. Y. Lee, J. Kim, J. Yeo, and H. Kang, "Can shortterm constraint-induced movement therapy combined with visual biofeedback training improve hemiplegic upper limb function of subacute stroke patients?" Annals of Rehabilitation Medicine, vol. 40, no. 6, pp. 998-1009, 2016.

[4] K. Corti. (2006) Games-based learning; a serious business application. [Online]. Available: https: //www.cs.auckland.ac.nz/courses/compsci777s2c/lectures/ Ian/serious\%20games\%20business\%20applications.pdf

[5] D. Charsky, "From edutainment to serious games: A change in the use of game characteristics," Games and culture, vol. 5, no. 2, pp. 177-198, 2010.

[6] R. W. Picard, “Affective computing,” MIT Press, 1997.

[7] J. Tao and T. Tan, "Affective computing: A review," in International Conference on Affective computing and intelligent interaction. Springer, 2005, pp. 981-995.

[8] T. S. Saini, M. Bedekar, and S. Zahoor, "Analysing human feelings by affective computing-survey," in Computing Communication Control and automation (ICCUBEA), 2016 International Conference on. IEEE, 2016, pp. 1-6.

[9] D. Webster and O. Celik, "Systematic review of kinect applications in elderly care and stroke rehabilitation," Journal of neuroengineering and rehabilitation, vol. 11, no. 1, p. 108, 2014.

[10] H. Sveistrup, "Motor rehabilitation using virtual reality," Journal of neuroengineering and rehabilitation, vol. 1, no. 1, p. 10, 2004.

[11] M. Osumi, A. Ichinose, M. Sumitani, N. Wake, Y. Sano, A. Yozu, S. Kumagaya, Y. Kuniyoshi, and S. Morioka, "Restoring movement representation and alleviating phantom limb pain through short-term neurorehabilitation with a virtual reality system," European Journal of Pain, vol. 21, no. 1, pp. 140-147, 2017.

[12] K. Lohse, N. Shirzad, A. Verster, N. Hodges, and H. M. Van der Loos, "Video games and rehabilitation: using design principles to enhance engagement in physical therapy," Journal of Neurologic Physical Therapy, vol. 37, no. 4, pp. 166-175, 2013.
[13] D. Tinson, "How stroke patients spend their days: an observational study of the treatment regime offered to patients in hospital with movement disorders following stroke," International Disability Studies, vol. 11, no. 1, pp. 45-49, 1989.

[14] V. Gadidi, M. Katz-Leurer, E. Carmeli, and N. M. Bornstein, "Long-term outcome poststroke: predictors of activity limitation and participation restriction," Archives of physical medicine and rehabilitation, vol. 92, no. 11, pp. 1802-1808, 2011.

[15] T. V. Veenith, A. H. Din, D. M. Eaton, and R. M. Burnstein, "Perioperative care of a patient with stroke," International archives of medicine, vol. 3, no. 1, p. 33, 2010.

[16] P. Langhorne, R. Wagenaar, and C. Partridge, "Physiotherapy after stroke: more is better?" Physiotherapy Research International, vol. 1, no. 2, pp. 75-88, 1996.

[17] R. W. Teasell, N. C. Foley, S. K. Bhogal, and M. R. Speechley, "An evidence-based review of stroke rehabilitation," Topics in stroke Rehabilitation, vol. 10, no. 1, pp. 29-58, 2003.

[18] G. J. Barton, M. B. Hawken, R. J. Foster, G. Holmes, and P. B. Butler, "The effects of virtual reality game training on trunk to pelvis coupling in a child with cerebral palsy," Journal of neuroengineering and rehabilitation, vol. 10, no. 1, p. 15, 2013.

[19] G. C. Burdea, D. Cioi, A. Kale, W. E. Janes, S. A. Ross, and J. R. Engsberg, "Robotics and gaming to improve ankle strength, motor control, and function in children with cerebral palsy-a case study series," IEEE Transactions on Neural Systems and Rehabilitation Engineering, vol. 21, no. 2, pp. 165-173, 2013.

[20] L. Mitchell, J. Ziviani, S. Oftedal, and R. Boyd, "The effect of virtual reality interventions on physical activity in children and adolescents with early brain injuries including cerebral palsy," Developmental Medicine \& Child Neurology, vol. 54, no. 7, pp. 667-671, 2012.

[21] R. Riener, E. Dislaki, U. Keller, A. Koenig, H. Van Hedel, and A. Nagle, "Virtual reality aided training of combined arm and leg movements of children with cp." Studies in health technology and informatics, vol. 184, pp. 349-355, 2012.

[22] C. Bryanton, J. Bosse, M. Brien, J. Mclean, A. McCormick, and H. Sveistrup, "Feasibility, motivation, and selective motor control: virtual reality compared to conventional home exercise in children with cerebral palsy," Cyberpsychology \& behavior, vol. 9, no. 2, pp. 123-128, 2006.

[23] A. Henderson, N. Korner-Bitensky, and M. Levin, "Virtual reality in stroke rehabilitation: a systematic review of its effectiveness for upper limb motor recovery," Topics in stroke rehabilitation, vol. 14, no. 2, pp. 52-61, 2007.

[24] M. Mat Rosly, H. Mat Rosly, G. M. Davis OAM, R. Husain, and N. Hasnan, "Exergaming for individuals with neurological disability: a systematic review," Disability and rehabilitation, pp. 1-9, 2016.

[25] T. Massetti, I. L. Trevizan, C. Arab, F. M. Favero, D. C. Ribeiro-Papa, and C. B. de Mello Monteiro, "Virtual reality in multiple sclerosis-a systematic review," Multiple Sclerosis and Related Disorders, vol. 8, pp. 107-112, 2016. 
[26] B. C. Smits-Engelsman, L. D. Jelsma, and G. D. Ferguson, "The effect of exergames on functional strength, anaerobic fitness, balance and agility in children with and without motor coordination difficulties living in low-income communities," Human Movement Science, 2016.

[27] M. Ulicsak, Games in education: serious games: A Futurelab literature review. FutureLab, 2010.

[28] T. Susi, M. Johannesson, and P. Backlund, "Serious games: An overview," Institutionen för kommunikation och information, 2007.

[29] D. Druckman, "The educational effectiveness of interactive games," Simulation and gaming across disciplines and cultures: ISAGA at a watershed, pp. 178-187, 1995.

[30] H. W. Giessen, "Serious games effects: an overview," Procedia-Social and Behavioral Sciences, vol. 174, pp. 2240 2244, 2015.

[31] M. Griffiths, "The therapeutic use of videogames in childhood and adolescence," Clinical child psychology and psychiatry, vol. 8, no. 4, pp. 547-554, 2003.

[32] J.-H. Annema, M. Verstraete, V. V. Abeele, S. Desmet, and D. Geerts, "Video games in therapy: a therapist's perspective," International Journal of Arts and Technology, vol. 6, no. 1, pp. 106-122, 2012.

[33] J. Halton, "Virtual rehabilitation with video games: A new frontier for occupational therapy," Occupational Therapy Now, vol. 9, no. 6, pp. 12-14, 2008.

[34] S. V. Adamovich, G. G. Fluet, E. Tunik, and A. S. Merians, "Sensorimotor training in virtual reality: a review," NeuroRehabilitation, vol. 25, no. 1, pp. 29-44, 2009.

[35] D. Jack, R. Boian, A. S. Merians, M. Tremaine, G. C. Burdea, S. V. Adamovich, M. Recce, and H. Poizner, "Virtual realityenhanced stroke rehabilitation," IEEE transactions on neural systems and rehabilitation engineering, vol. 9, no. 3, pp. 308318, 2001.

[36] A. Li, Z. Montaño, V. J. Chen, and J. I. Gold, "Virtual reality and pain management: current trends and future directions," Pain, vol. 1, no. 2, pp. 147-157, 2011.

[37] W. Liu, "Natural user interface-next mainstream product user interface," in Computer-Aided Industrial Design \& Conceptual Design (CAIDCD), 2010 IEEE 11th International Conference on, vol. 1. IEEE, 2010, pp. 203-205.

[38] C.-Y. Chang, B. Lange, M. Zhang, S. Koenig, P. Requejo, N. Somboon, A. A. Sawchuk, and A. A. Rizzo, "Towards pervasive physical rehabilitation using microsoft kinect," in Pervasive Computing Technologies for Healthcare (PervasiveHealth), 2012 6th International Conference on. IEEE, 2012, pp. 159-162.

[39] E. Pietroni, A. Pagano, and C. Rufa, "The etruscanning project: gesture-based interaction and user experience in the virtual reconstruction of the regolini-galassi tomb," in Digital Heritage International Congress (DigitalHeritage), 2013, vol. 2. IEEE, 2013, pp. 653-660.

[40] M. Dontschewa, S. Rosmann, and M. Marinov, "Using motion capturing sensor systems for natural user interface," in Scientific Conference Electronics (ET), International. IEEE, 2016, pp. 1-4.
[41] A. K. Roy, Y. Soni, and S. Dubey, "Enhancing effectiveness of motor rehabilitation using kinect motion sensing technology," in Global Humanitarian Technology Conference: South Asia Satellite (GHTC-SAS), 2013 IEEE. IEEE, 2013, pp. 298304.

[42] K. Morrow, C. Docan, G. Burdea, and A. Merians, "Lowcost virtual rehabilitation of the hand for patients post-stroke," in Virtual Rehabilitation, 2006 International Workshop on. IEEE, 2006, pp. 6-10.

[43] C. Rodriguez-de Pablo, S. Balasubramanian, A. Savić, T. D. Tomić, L. Konstantinović, and T. Keller, "Validating armassist assessment as outcome measure in upper-limb post-stroke telerehabilitation," in Engineering in Medicine and Biology Society (EMBC), 2015 37th Annual International Conference of the IEEE. IEEE, 2015, pp. 4623-4626.

[44] M. Ma and K. Bechkoum, "Serious games for movement therapy after stroke," in Systems, Man and Cybernetics, 2008. SMC 2008. IEEE International Conference on. IEEE, 2008, pp. 1872-1877.

[45] Unity. (2017) Personal edition. [Online]. Available: https: //unity3d.com/pt/unity/personal-edition

[46] A. Bandura, "Guide for constructing self-efficacy scales," Self-efficacy beliefs of adolescents, vol. 5, no. 307-337, 2006.

[47] Kinect. (2017) Kinect sensor for xbox one. [Online]. Available: http://www.xbox.com/xbox-one/accessories/kinect

[48] Affectiva. (2017) Emotion recognition software and analysis. [Online]. Available: http://www.affectiva.com/

[49] L. Motion. (2017) Leap motion. [Online]. Available: https://www.leapmotion.com/

[50] M. Khademi, H. Mousavi Hondori, A. McKenzie, L. Dodakian, C. V. Lopes, and S. C. Cramer, "Free-hand interaction with leap motion controller for stroke rehabilitation," in Proceedings of the extended abstracts of the 32nd annual ACM conference on Human factors in computing systems. ACM, 2014, pp. 1663-1668.

[51] Z.-r. Wang, P. Wang, L. Xing, L.-p. Mei, J. Zhao, T. Zhang et al., "Leap motion-based virtual reality training for improving motor functional recovery of upper limbs and neural reorganization in subacute stroke patients," Neural regeneration research, vol. 12, no. 11, p. 1823, 2017.

[52] T. Pascu, M. White, and Z. Patoli, "Motion capture and activity tracking using smartphone-driven body sensor networks," in Innovative Computing Technology (INTECH), 2013 Third International Conference on. IEEE, 2013, pp. 456-462.

[53] ManoMotion. (2017) Manomotion. [Online]. Available: https://www.manomotion.com/ 